\title{
Atrioventricular block occurring several months after radiofrequency ablation for the treatment of atrioventricular nodal re-entrant tachycardia
}

\author{
O Elhag, H C Miller
}

\begin{abstract}
Atrioventricular (AV) block following radiofrequency (RF) ablation for the treatment of AV nodal re-entrant tachycardia (AVNRT) is a rare but well recognised complication of the procedure-the reported incidence ranges from $1 \%$ to $21 \%$. Almost all cases of AV block occur during or shortly after the procedure, are transient, and recover quickly. Two patients (a 22 year man and a 72 year old woman) with symptomatic AV block occurring several months after slow pathway RF ablation, requiring permanent pacemaker implantation, are described. Both patients had had several 24 hour Holter recordings before the procedure, and in neither case was there any evidence of intermittent or persistent AV block. This is a rare complication with no definitive predictors; however, all efforts should be made to exclude AV block in patients presenting with suggestive symptoms following RF ablation. With the wide use of RF ablation for the treatment of AVNRT, more cases are likely to occur. A registry should allow documentation of the incidence of this complication.

(Heart 1998;79:616-618)
\end{abstract}

Keywords: atrioventricular nodal re-entrant tachycardia; radiofrequency ablation; atrioventricular block; pacing

Atrioventricular (AV) block following radiofrequency (RF) ablation for the treatment of AV nodal re-entrant tachycardia (AVNRT) is a rare but well recognised complication of the procedure. ${ }^{1}$ The reported incidence in the literature ranges from $1 \%$ to $21 \% .^{23}$ Almost all cases of AV block occur during or shortly after the procedure, are transient, and recover quickly. Persistent AV block is very rare and only a few cases require the implantation of a permanent pacemaker. ${ }^{45}$

We describe two cases of symptomatic AV block occurring several months after slow pathway $\mathrm{RF}$ ablation, requiring permanent pacemaker implantation. Both patients had had several 24 hour Holter recordings before the procedure, and in neither case was there any evidence of intermittent or persistent AV block.

\section{Case 1}

A 22 year old man was first referred in June 1994 for consideration of RF ablation of troublesome tachycardia. His symptoms had started a few years earlier and had required several admissions to hospital despite treatment with various antiarrhythmic drugs. His symptoms were typically fast palpitation associated with breathlessness, chest tightness, and profuse sweating. He had once lost consciousness during tachycardia but recovered fully after a few minutes. ECG during symptoms showed a narrow QRS complex tachycardia at 240 beats/min.

In December 1994 electrophysiology study and RF ablation were done under light sedation. Catheters were positioned in the high right atrium, right ventricular apex, the coronary sinus, and across the tricuspid valve to record His bundle electrograms. At baseline $\mathrm{A}-\mathrm{H}$ and $\mathrm{H}-\mathrm{V}$ intervals were within the normal range. Dual AV nodal physiology was demonstrated with a $110 \mathrm{~ms}$ jump in A-H interval for a $20 \mathrm{~ms}$ decrement in coupling interval using extrastimulus technique. Typical AVNRT with cycle length of $280 \mathrm{~ms}$ was readily inducible following the administration of isoprenaline. Radiofrequency energy was delivered in the posterior AV junction using an anatomical approach and attempts to identify slow pathway potentials were not made. An A:V ratio of 0.1:0.5 was considered optimal for RF ablation. Following unsuccessful ablation a more anterior site was chosen as described by Jazayeri et al. ${ }^{3}$ Three RF ablations were given. Junctional rhythm with occasional ventriculoatrial (VA) block occurred with each energy application; no antegrade block occurred. Following the third energy delivery there was complete ablation of the slow pathway with no change in A-H or $\mathrm{H}-\mathrm{V}$ intervals from baseline. Tachycardia was non-inducible with and without isoprenaline. The patient was observed overnight and discharged the following day. The ECG remained normal throughout.

At follow up a month later the patient was well with no recurrence of tachycardia. $\mathrm{He}$
Dr Elhag.

Accepted for publication 27 November 1997 
described missed beats and 24 hour Holter monitoring showed conducted supraventricular extrasystoles correlating with symptoms. He was reassured and discharged from follow up. Six months after RF ablation he was referred with chest pain and lightheadedness. Twenty four hour Holter monitoring showed type I second degree AV block during the night and frequent supraventricular extrasystoles. $\mathrm{He}$ continued to have symptoms, and repeated 24 Holter recordings and a cardiac event recorder used over three weeks failed to reveal significant abnormalities. Nine months after RF ablation he had an episode of syncope and 24 hour Holter recording showed frequent episodes of 2:1 AV block with symptoms. A dual chamber pacemaker was implanted with no recurrence of symptoms.

\section{Case 2}

A 72 year old woman had troublesome paroxysmal supraventricular tachycardia for over 25 years. Symptoms had been reasonably controlled with various antiarrhythmic drugs.

In September 1993 she was admitted to hospital with more frequent symptoms and the development of presyncope and syncope during tachycardia as a new symptom. ECG on admission showed a narrow QRS tachycardia at 210 beats $/ \mathrm{min}$. She had no organic heart disease.

An electrophysiology study and RF ablation were performed in November 1993. A similar protocol as in case 1 was followed. The A-H and $\mathrm{H}-\mathrm{V}$ intervals were normal at baseline and typical AVNRT was induced. Two RF deliveries were required to achieve complete slow pathway ablation after which tachycardia could not be induced with or without isoprenaline.

Accelerated junctional rhythm developed during the second RF ablation with occasional VA block. Following successful ablation A-H remained unchanged from baseline. Shortly after ablation the patient developed atrial fibrillation and she was monitored for 48 hours before sinus rhythm returned spontaneously. The ECG was normal on discharge on the third day after ablation. A month later, ECG showed first degree AV block with a PR interval of 0.22 seconds, but she was completely asymptomatic. This did not change over the following month and she was discharged from follow up.

Fifteen months after ablation she was referred with palpitations suggestive of extrasystoles. Resting ECG showed first degree AV block as before but 24 hour tape recording showed frequent episodes of 2:1 AV block. An episode of syncope occurred a few days later and a dual chamber pacemaker was subsequently implanted with no recurrence of symptoms.

\section{Discussion}

To our knowledge these are the first cases of symptomatic AV block occurring many months after RF ablation. Several reports of delayed (up to one month) AV block have suggested that the occurrence of transient AV block during the procedure is a strong marker for the late development of AV block with a predictive value of about $20 \% .{ }^{67}$ This complication did not occur in our patients; however, VA block during ectopic junctional tachycardia occurred during ablation in both cases. This was manifest by occasional failure of single junctional beats to conduct back to the atrium. The junctional rhythm during ablation in both cases was irregular with a cycle length varying between 340 and $500 \mathrm{~ms}$. VA block in these circumstances could be caused by refractory fast pathway conduction. No complete VA dissociation or prolonged periods of VA block occurred. Although the predictive value of VA block during ectopic junctional tachycardia for the development of AV block is low, this seems to be the only predictor in our patients. ${ }^{89}$

Hintringer et al suggested that an early A potential on the ablating catheter A (Md) compared to the A potential recorded in the proximal His $A(H)$ is a predictor for complete AV block. In their series they identified an interval of less than $20 \mathrm{~ms}$ to be associated with the development of AV block. The A $(\mathrm{H})$ to $\mathrm{A}$ (Md) interval was measured as $30 \mathrm{~ms}$ and $25 \mathrm{~ms}$ in cases 1 and 2 , respectively. ${ }^{8}$

A possible explanation for the development of symptomatic heart block several months after successful RF ablation is the continuing healing process of the RF lesion. The lesion is a well demarcated area of coagulative necrosis surrounded by inflammatory cell infiltrate and haemorrhage. Fibrosis in this peripheral zone may lead to extension of the lesion and impairment of conduction, with resulting advanced AV block. ${ }^{10} 11$

Another possible explanation for the late development of $\mathrm{AV}$ block in these patients is extension of microvascular endothelial cell injury. Nath et al showed that tissues next to the site of RF ablation show evidence of thermal injury, which might progress to cause late electrophysiological phenomena. ${ }^{12}$ Resolution of the same process might explain recurrence of conduction in accessory pathways that initially appeared to have been successfully ablated. ${ }^{13}$

For practical purposes, we consider this complication rare and, as yet, no definitive predictors exist. However, all efforts should be made to exclude AV block in patients presenting with suggestive symptoms following RF ablation. With the wide use of RF ablation for the treatment of AVNRT, more cases are likely to occur. A registry ${ }^{5}$ should allow documentation of the incidence of this complication.

1 Chen S-A, Chiang C-E, Tai C-T, et al. Complications of diagnostic electrophysiologic studies and radiofrequency catheter ablation in patients with tachyarrhythmias: an eight-year survey of 3,900 consecutive procedures in a tertiary referral center. Am f Cardiol 1996;77:41-6.

2 Scheinman MM. NASPE survey on catheter ablation. PACE 1995;18:1474-8

3 Jazayeri MR, Hempe SL, Sra JS, et al. Selective transcatheter ablation of the fast and slow pathways using radiofrequency energy in patients with atrioventricular nodal re-entrant tachycardia. Circulation 1992;85:1318-28.

4 Fujimura O, Schoen J, Kuo C-S, et al. Delayed recurrence of atrioventricular block after radiofrequency ablation of atrioventricular node re-entry: a word of caution. Am Heart f 1993;125:901-4.

5 Schaffer MS, Silka MJ, Ross BA, et al. Inadvertent atrioventricular block during radiofrequency catheter ablation. Results of the pediatric radiofrequency ablation registry. Circulation 1996;94:3214-20. 
6 Fenelon G, d'Avila A, Malacky T, et al. Prognostic significance of transient complete atrioventricular block during radiofrequency ablation of atrioventricular node re-entrant tachycardia. Am f Cardiol 1995; 75:687-702

7 Goyal R, Langberg JJ, Kim Y-N, et al. Complete atrioventricular (AV) block complicating radiofrequency catheter modification of AV nodal re-entrant tachycardia (AVNRT) [abstract]. PACE 1991;14(suppl II):658

8 Thakur RK, Klein GJ, Yee R, et al. Junctional tachycardia: a useful marker during radiofrequency ablation for atrioventricular node re-entrant tachycardia. $\mathcal{f} \mathrm{Am}$ Coll Cardiol 1993;22:1706-10.

9 Hintringer F, Hartikainen J, Wyn Davies D, et al. Prediction of atrioventricular lock during radiofrequency ablation of the slow pathway of the atrioventricular node. Circulation $1995 ; 92 \cdot 3490-6$
10 Fenelon G, Brugada P. Delayed effects of radiofrequency energy: mechanisms and clinical implications. PACE 1996; 19:484-9.

11 Simmers TA, Wever EFD, Wittkampf FHM, et al. Change in delay of atrioventricular conduction after radiofrequency catheter ablation for atrioventricular nodal re-entry tachycardia. Br Heart f 1995;73:442-4.

12 Nath S, Whayne JG, Kaul S, et al. Effects of radiofrequency catheter ablation on regional myocardial blood flow. Possible mechanism for late electrophysiological outcome. Circulation 1994;89:2667-72

13 Engelstein ED, Kunze KP, Duckeck W. Radiofrequency current modulation of atrioventricular nodal conduction: implications of unintentional third-degree conduction block [abstract]. PACE 1993;16(Pt I):1001-5.

\section{IMAGES IN CARDIOLOGY}

\section{Rapid progression of pericardial malignant mesothelioma}
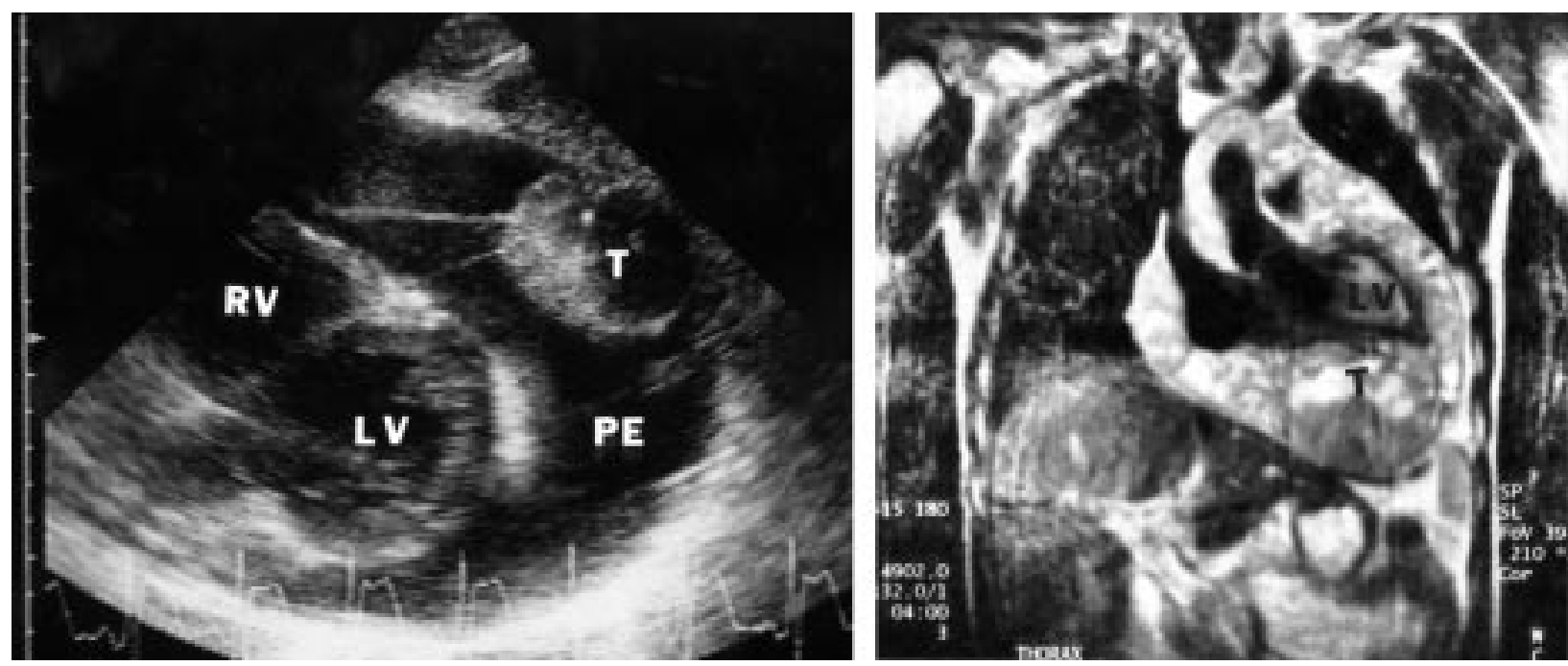

A 19 year old man with no history of cardiac disease had been admitted to another hospital six months previously with progressively worsening breathlessness and a decrease in exercise tolerance. Chest radiography showed cardiomegaly and echocardiography showed pericardial effusion, moderate ventricular function, no valve dysfunction, and a bad collapsing inferior vena cava. Although malignancy was suspected, this could not be confirmed by either echocardiography (abdomen) or computed tomography of the chest and abdomen. Pericardiocentesis released two litres of clean fluid that contained only inflammatory cells. Despite repeated echocardiography no recurrent pericardial effusion was seen.

After six months he returned with massive pericardial effusion. On examination in our hospital he was unwell, short of breath, had a regular pulse, and no pulses paradoxes. There were signs of cardiomegaly, normal heart sounds and systolic murmur. Echocardiography showed massive pericardial effusion and a solid structure at the apex and the left posterior ventricular wall. Both ventricles were compressed. Magnetic resonance imaging showed massive myocardial infiltration with a large mass infiltrating into the posterior left ventricular wall $(10 \times 7 \times 7 \mathrm{~cm})$. There was also infiltration of the mediastinum and around the arcus aorta. Besides mild pleural effusion, no other (thoracic or

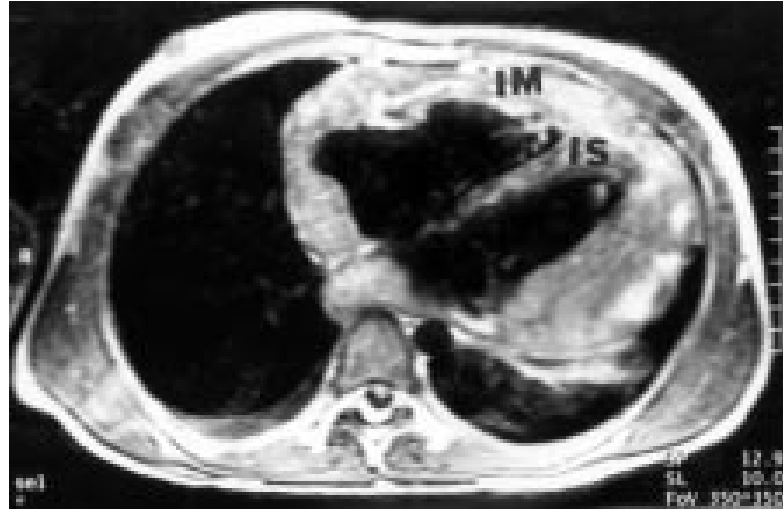

A small lateral thoracotomy was performed and tissue was obtained. Histological examination revealed a primary malignant pericardial tumour, probably mesothelioma. Within six months of the first evidence for pericardial effusion the patient died from inflow restriction. (IS, interventricular septum; IM, infiltrating mass; LV, left ventricle; PE, pericardial effusion; RV, right ventricle; $T$, tumour.)

G TJEERDSMA J BROUWER

D J VAN VELDHUISEN 